\title{
Finite Element Analysis of Elastic-Plastic Contact Mechanic Considering the Effect of Contact Geometry and Material Properties
}

\begin{abstract}
Abodol Rasoul Sohouli ${ }^{*}$, Ali Maozemi Goudarzi, Reza Akbari Alashti
Department of Mechanical Engineering, Babol Noshirvani University of Technology, Babol. Email: *Rasoul.sohouli@gmail.com

Received September $1^{\text {st }}, 2011$; revised October $3^{\text {rd }}, 2011$; accepted October $11^{\text {th }}, 2011$.

ABSTRACT

Each surface of roughness has different shape of asperity which is modeled with various shapes of analytical models. In this paper, the differences among various models of shape of asperity investigate using the Finite Element Method (FEM) and various analytical models. The contact stresses in rough surfaces are calculated analytically using various asperity shape models. Finite element analysis is also carried out assuming three types of material properties namely, the linear, the elastic-perfect plastic and the elastic-nonlinear hardening. The analytical results are compared with the results obtained by the finite element method. The results illustrate for using a deterministic approach which the numerical models are suitable. In hertz model, the result of force is very big in interface of causing deformation plastic, while Model Zhao has almost same result with FEM nonlinear property model. It is observed that the results obtained from Zhao's model are generally in a better agreement with the results obtained from various finite element models especially in elastic-plastic and plastic zones, hence it may be concluded that Zhao's model can be used for analyzing the rough surfaces in contact mechanics.
\end{abstract}

Keywords: Contact Mechanics; Micro-Contact; Finite Element Method (FEM); Zhao's Model; Chang's Model

\section{Introduction}

The rough contact problem has been studied for many years as it is critically important to understand the tribological phenomenon such as friction, wear, contact fatigue, and sealing. Engineering surfaces have roughness and even highly polished surfaces possess some degrees of Roughness. When two engineering surfaces are pressed together, contact occurs at the peaks of the Surfaces where the contact pressure and subsurface stress can be extremely high, often causing plastic deformation of these spots. The contact between a deformable half-space and a rigid sphere was first solved by Hertz in 1896 [1] but this model only considers the elastic contact and disdains the effects of the roughness and the effects of the plasticity, and the real contact area is unvalued.

Greenwood and Williamson [2] pioneered the study of frictionless contact between a hemisphere and a rigid flat (the GW model) applied the Hertz contact solution to model an entire contact surface of elastic asperities. To supplement the GW model, many elastic-plastic asperity models have been devised.
The study of the deformation behavior of contact asperities and the accurate modeling of rough surfaces is important for understanding contact problems. Several theories can be applied to deal with the microcontacts of two contact surfaces [3-9].

Some new models were proposed to consider elasticplastic contact that Chang's model [10] and Zhao's Model [11] are investigated in this paper.

In this work, some selected models are in particular reported, whose formulas, have been used to test the different models of roughness description developed in [12, 13]. The calculated contact zones and loads are compared with different numerical models.

The results illustrate for using a deterministic approach which the numerical models are suitable.

\section{Models of Contact Mechanics}

The contact model was first established by Heinrick Hertz in 1882, although the model considered the elastic contact only and the effects of the surface roughness and plasticity were not considered. Contact problems with rough surfaces have been modeled with stochastic techniques. 
The pioneering contribution to this field was done by Greenwood and Williamson, who developed a basic elastic contact model (GW model) [2]. The basic asperity GW model has been extended to other models, two of which are explained below.

\subsection{Chang's Model}

Chang's model or CEB model is an elastoplastic based model. This model is similar to the Greenwood and Williamson model but it considers the volume conservation [10]. Behavior of the contact is related to the interference, $\delta$, between the two surfaces. If $\delta$ is smaller than it's critical value $\delta$ c, i.e. $\delta<\delta_{c}$, the contact is assumed to be elastic, otherwise the contact is assumed to be plastic. The relations used for the elastic regime are based on the Hertz theory. Therefore, the contact area, Ae, and the elastic contact load, fe, are calculated as:

$$
\begin{aligned}
& A_{e}=\pi a_{e}^{2}=\pi R \delta \\
& a_{e}=(R \delta)^{1 / 2} \\
& f_{e}=\frac{4}{3} E^{*} R^{1 / 2} \delta^{3 / 2}
\end{aligned}
$$

where $R$ and $a_{e}$ are the radius of the sphere and the radius of the circular contact area respectively and $E^{*}$ is the composite elasticity modulus which is expressed as follow:

$$
E^{*}=\left(\frac{1-v_{1}^{2}}{E_{1}}+\frac{1-v_{2}^{2}}{E_{2}}\right)^{-1}
$$

the critical interference, $\delta_{c}$, is introduced by the expression

$$
\delta_{c}=R\left(\frac{\pi k H}{2 E^{*}}\right)
$$

where the interference is, $\delta \geq \delta_{c}{ }^{2}$, the relations are found to be.

$$
\begin{aligned}
& A_{p}=\pi R \delta\left(2-\frac{\delta_{c}}{\delta}\right) \\
& a_{p}=\left(R \delta\left(2-\frac{\delta_{c}}{\delta}\right)\right)^{1 / 2}
\end{aligned}
$$

The contact load for each asperity under the plastic conditions is:

$$
f_{p}=A_{p} k H
$$

This model is only used for fully elastic and fully plastic conditions and there are no relations developed for the elastic-plastic regime.

\subsection{Zhao's Model}

This model is an elastic-plastic asperity contact model for rough surfaces introduced by Zhao et al. [11]. In Zhao's model three regions of fully elastic, elastic plastic and fully plastic contacts are considered. In the elastic region, where $\delta<\delta_{c}$, the governing relations are similar to those of CEB model. When the interference, $\delta$, between the two surfaces is increased i.e. $\delta \geq 54 \delta_{c}$, the following equation is employed for calculation of the radius of fully plastic contact.

$$
a_{p}=(2 R \delta)^{1 / 2}
$$

Therefore, the surface of the contact area is:

$$
A_{p}=\pi a_{p}^{2}=2 \pi R \delta
$$

Hence, the contact load for each asperity is found to be:

$$
f_{p}=A_{p} H=2 \pi R \delta H
$$

In the elastic-plastic region, where $\delta_{c} \leq \delta<54 \delta_{c}$, the relation between the mean pressure $\left(P_{m}\right)$ and the interference $(\delta)$, in order to calculate the area of the contact and the elastoplastic load is as follow:

$$
P_{m}=a_{1}+a_{2} \ln \left(\frac{\delta}{r}\right)
$$

where $a_{1}$ and $a_{2}$ are two constants to be determined and $r$ is the contact radius of the asperity.

Finally, the following equations are used for the radius, $R$, area of the contact surface, $A_{e p}$, and the contact load, $f_{e p}$, respectively:

$$
\begin{aligned}
A_{e p} & =\pi R \delta\left[1-2\left(\frac{\delta-\delta_{c}}{53 \delta_{c}}\right)^{3}+3\left(\frac{\delta-\delta_{c}}{53 \delta_{c}}\right)^{2}\right] \\
a_{e p} & =\left\{R \delta\left[1-2\left(\frac{\delta-\delta_{c}}{53 \delta_{c}}\right)^{3}+3\left(\frac{\delta-\delta_{c}}{53 \delta_{c}}\right)^{2}\right]\right\}^{1 / 2} \\
f_{e p} & =A_{e p}\left[H-H\left(1-\frac{2}{3} k\right) \frac{\ln 54 \delta_{c}-\ln \delta}{\ln 53}\right]
\end{aligned}
$$

\section{Finite Element Model}

The asperity is modeled in three geometrical shapes i.e. hemispherical, spherical and conical shapes against a rigid flat punch. All the three geometrical shapes have the same radius of $0.0005 \mathrm{~m}$ on the peak. In this analysis, axisymmetric 2-D models are used. A total of 11200 four-node bilinear axisymmetric elements were used to model each shape. The meshing of the model is refined near the region of contact in order to allow the curvature to be captured and accurately simulated during deforma- 
tion. The contact force acting on each model is found from the reaction forces at the corresponding base nodes that retain the desired interference. The material property is modeled in two types, elastic-perfect plastic and nonlinear hardening. The elastic perfect plastic material is assumed to have yield stress, $\sigma_{Y}$, equal to $24.4 \mathrm{M} \mathrm{Pa}$. The stress-strain relation of the elastic-nonlinear hardening material is shown in Figure 1.

A reference point is defined for the rigid flat punch and is allowed to move down as shown in Figure 2. The

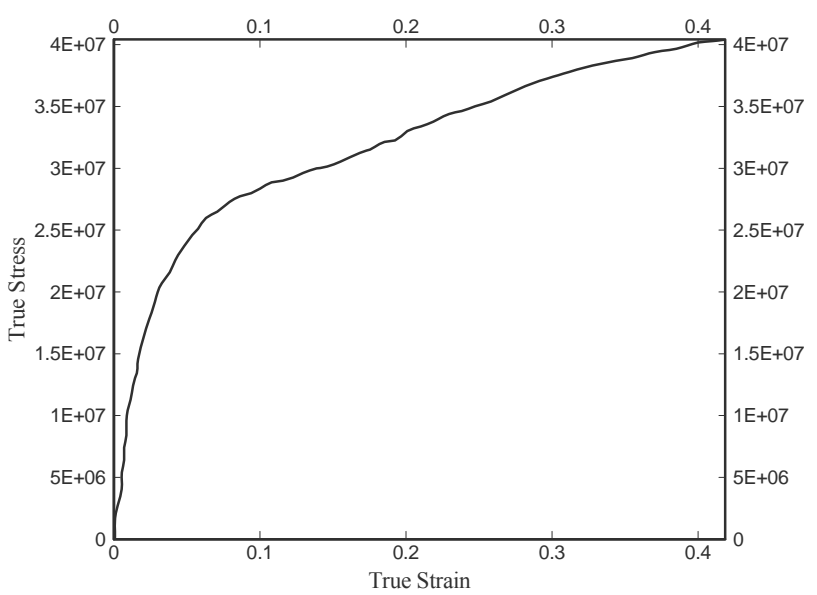

Figure 1. Nonlinear behavior property of material in ABAQUS.
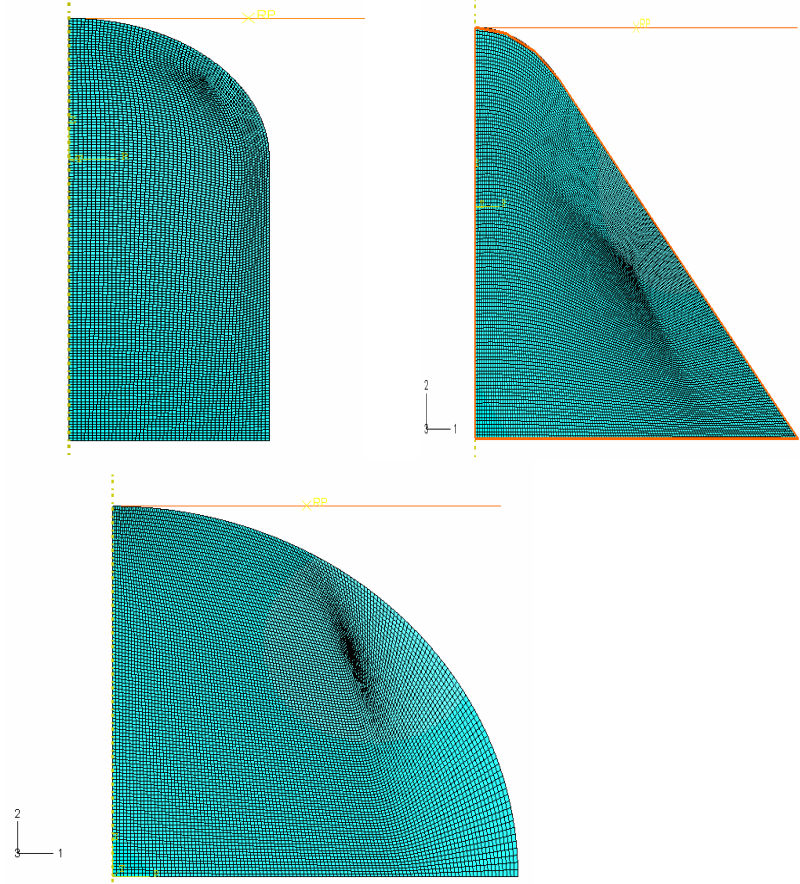

Figure 2. Finite element modeling of various shapes of asperity. nodes at the base of all three models are fixed in all directions. The contact force acting on the model is found from the reaction forces on the reference point that retain the desired interference. Finite element meshes of the three model generated by ABAQUS are shown in Figure 2.

\section{Results and Discussion}

The results of the finite element models are presented for a variety of interferences. The contact area and the interference are normalized by the mean area of $0.785 \mathrm{E}-6 \mathrm{~m}^{2}$ and critical interference of $0.462 \mathrm{E}-6 \mathrm{~m}$ respectively.

The contact forces obtained for all models are normalized by the Hertzian contact force of $0.011800474 \mathrm{~N}$ as obtained from the equation (3). In order to improve the computational efficiency, the radii of the asperity peaks are assumed to be constant. In Figure 3, the dimensionless contact force is plotted as a function of interfere.

At low interference ratios, the differences between the dimensionless contact forces in all modes are small because the contact is in the elastic state. It is interesting to note that the Chang's model predicts the least loads at big interferences. This is because of the fact that the Chang's model assumes that for all plastically deformed asperities the average pressure over the contact area is equal to $\mathrm{kH}$. However, in the Zhao's model, it is assumed that for all plastically deformed asperities the average pressure over the contact area is equal to $\mathrm{H}$, So the contact force for plastic zone predicted by the Zhao's model is bigger than that of the Chang's model. In the Zhao's model, transition from fully elastic deformation to fully plastic flow of the contacting asperity is modeled based on the contact mechanics theories in conjunction with the continuity and smoothness of variables across different modes of deformation. As shown in Fig. 3, the dimensionless contact force predicted by the Zhao's model is smoothly connected from the elastic state to the plastic state.

In Figure 4, the results of FEM analysis for the spherical asperity with both elastic-perfect plastic (EP PP) and elastic-nonlinear hardening properties (NLP) are compared with the Hertzian, Chang's model and Zhao's model. It is observed that the dimensionless contact force obtained by the FEM for both the elastic-perfect plastic and the elastic-nonlinear hardening materials follow the Zhao's model. Dimensionless contact forces for all three asperity shapes made of elastic-nonlinear hardening materials are shown and compared with three previous models in Figure 5. It is observed that the results obtained for both the spherical and conical asperity shapes follow the Zhao's model at all interferences while the result for the hemispherical shape follows the Chang's model. As can be seen from Figure 5 that the results obtained for the 


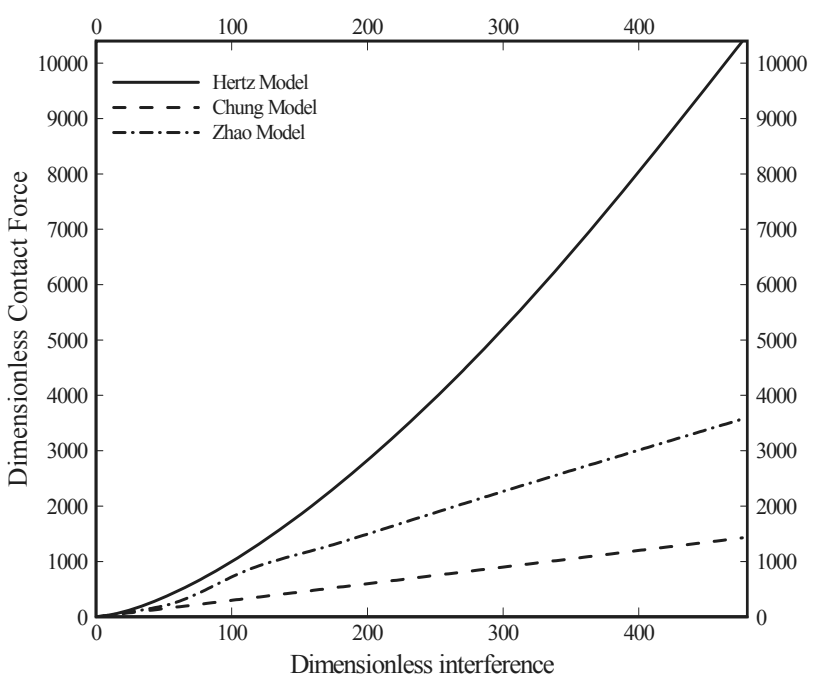

Figure 3. Dimensionless contact force versus interference for analytical models.

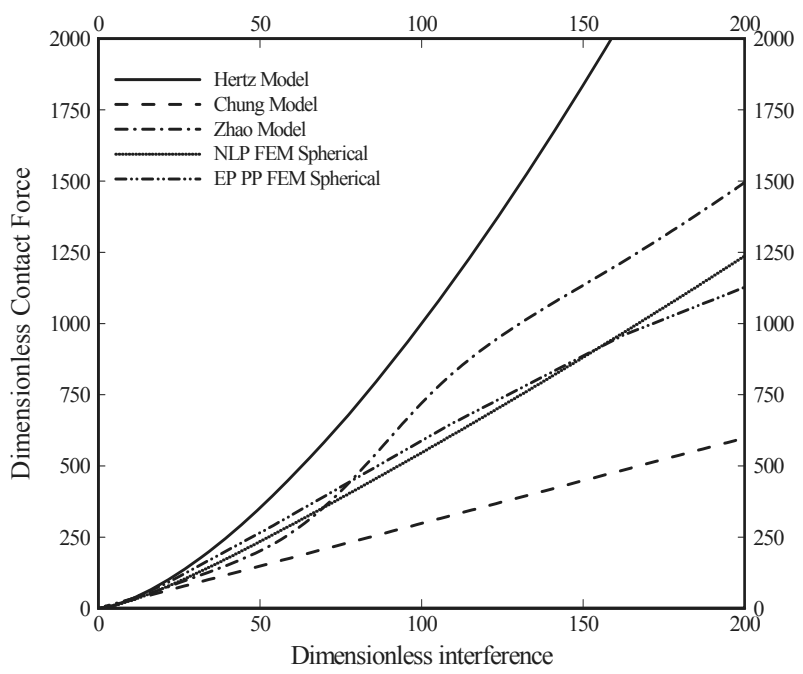

Figure 4. Dimensionless contact force versus interference for various models in medium interference.

spherical asperity shape follow the Zhao's model the best. It is due to the fact that relationships drive on base assumption spherical shape. The results obtained for all models are in good agreement with the Hertzian model for low interferences.

The results of dimensionless contact areas obtained for all three asperity shapes made of elastic - nonlinear hardening material properties and spherical shape made of elastic-perfect plastic material for a variety of interferences are shown in Figure 6.

\section{Conclusions}

This study showed that dimensionless contact force obtained

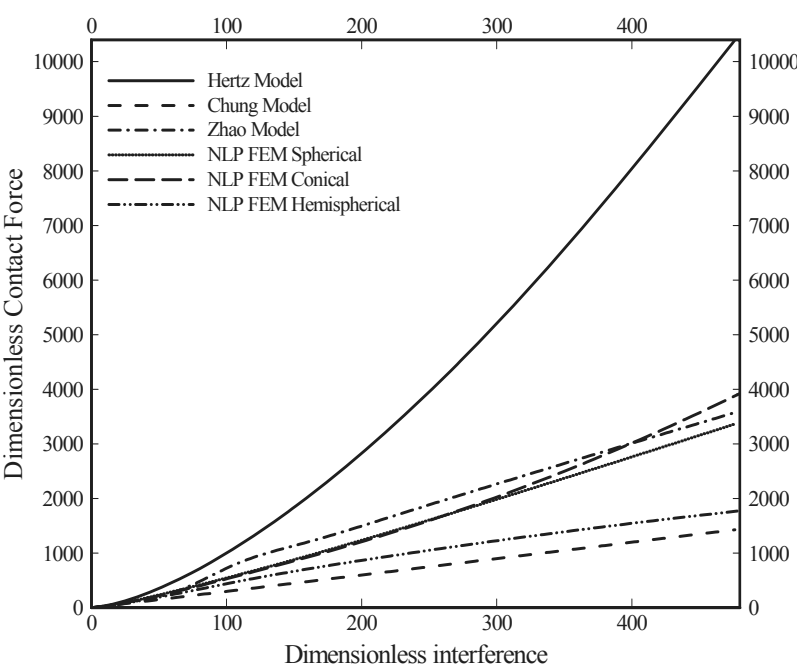

Figure 5. Dimensionless contact force versus interference for various models.

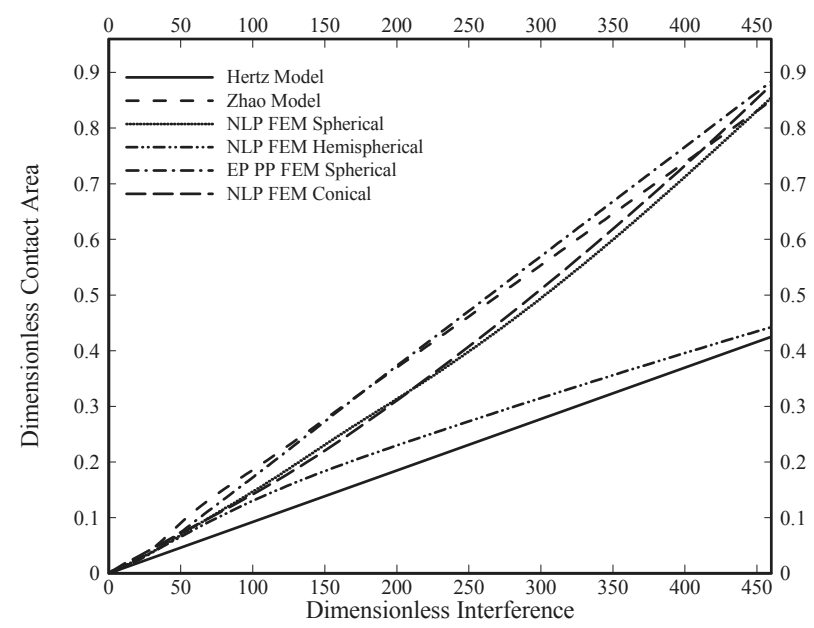

Figure 6. Dimensionless contact area versus interference for various models.

for various asperity shapes are in good agreement with Hertzian model for low interferences. However the finite element results obtained for spherical and hemispherical asperity shapes are in very good agreement with the Zhao's model for nearly the whole range of interferences.

It is observed that the results obtained the results obtained for both the spherical and conical asperity shapes follow the Zhao's model at all interferences while the result for the hemispherical shape follows the Chang's model but it can be said that Zhao's model are generally in a better agreement with the results obtained from various finite element models especially in elastic-plastic and plastic zones, hence it may be concluded that Zaho's model can be used for analyzing the rough surfaces in 
contact mechanics.

\section{REFERENCES}

[1] K. L. Johnson, "Contact Mechanics," Cambridge University Press, Cambridge, 1985.

[2] J. A. Greenwood and J. H. Tripp, "The Contact of Two Nominally Flat Rough Surfaces," Proceedings of the Institution of Mechanical Engineers, Vol. 185, No. 1, 1971, pp. 624-633. doi:10.1243/PIME_PROC_1970_185_069_02

[3] J. I. McCool, "Comparison of Model for Contact of Rough Surfaces," Wear, Vol. 107, No. 1, 1986, pp. 37-60. doi:10.1016/0043-1648(86)90045-1

[4] L. Kogut and I. Etsion, "Elastic-Plastic Contact Analysis of a Sphere and a Rigid Flat," Journal of Applied Mechanics, Vol. 69, 2002, pp. 657-662. doi: $10.1115 / 1.1490373$

[5] A. Majumdar and C. L. Tien, "Fractal Characterization and Simulation of Rough Surfaces," Wear, Vol. 136, No. 2, 1990, pp. 313-327. doi:10.1016/0043-1648(90)90154-3

[6] A. Majumdar and B. Bhushan, "Role of Fractal Geometry Inroughness Characterization and Contact Mechanics of Surfaces," Journal of Tribology, Vol. 112, No. 2, 1990, pp. 205-216. doi:10.1115/1.2920243

[7] A. Majumdar and B. Bhushan, "Fractal Model of ElasticPlastic Contact between Rough Surfaces," Journal of Tri- bology, Vol. 113, No. 1, 1991, pp. 1-11. doi:10.1115/1.2920588

[8] B. Bhushan and A. Majumdar, "Elastic-Plastic Contact for Bifractal Surfaces," Wear, Vol. 153, No. 1, 1992, pp. 53-64. doi:10.1016/0043-1648(92)90260-F

[9] C. Hardy, C. N. Baronet and G. V. Tordion, "The ElastoPlastic Indentation of a Half-Space by a Rigid Sp- here," International Journal for Numerical Methods in Engineering, Vol. 3, No. 4, 1971, pp. 451-462. doi:10.1002/nme.1620030402

[10] W. R. Chang, L. Etsion and D. B. Bogy, "An ElasticPlastic Model for the Contact of Rough Surfaces," Journal of Tribology, Vol. 109, 1987, pp. 257-263. doi:10.1115/1.3261348

[11] Y. Zhao and L. Chang, "A Model of Asperity Interactions In Elastic-Plastic Contact of Rough Surfaces," Journal of Tribology, Vol. 123, No. 4, 2001, pp. 857-864 doi:10.1115/1.1338482

[12] E. Ciulli, L. A. Ferreira, G. Pugliese and S. M. O. Tavares, "Rough Contacts Between Actual Engineering Surfaces Part I. Simple Models for Roughness Description," Wear, Vol. 264, No. 11-12, 2008, pp. 1105-1115. doi:10.1016/j.wear.2007.08.024

[13] G. Pugliese, S. M. O. Tavares, E. Ciulli and L. A. Ferreir, "Rough Contacts Between Actual Engineering Surfaces Part II. Contact Mechanics," Wear, Vol. 264, No. 11-12, 2008, pp. 1116-1128. doi:10.1016/j.wear.2007.08.027 